\title{
Left ventricular rotational abnormalities in hemophilia-insights from the three-dimensional speckle-tracking echocardiographic MAGYAR-Path Study
}

\author{
Attila Nemes ${ }^{1}$, Árpád Kormányos ${ }^{1}$, Péter Domsik ${ }^{1}$, Nóra Ambrus ${ }^{1}$, Nándor Gyenes ${ }^{1}$, Klára Vezendi ${ }^{2}$, \\ Imelda Marton ${ }^{2,3}$, Zita Borbényi ${ }^{3}$ \\ ${ }^{1}$ Department of Medicine, Faculty of Medicine, Albert Szent-Györgyi Clinical Center, University of Szeged, Szeged, Hungary; ${ }^{2}$ Department of \\ Transfusiology, Faculty of Medicine, Albert Szent-Györgyi Clinical Center, University of Szeged, Szeged, Hungary; ${ }^{3}$ Division of Haematology, \\ Department of Medicine, Faculty of Medicine, Albert Szent-Györgyi Clinical Center, University of Szeged, Szeged, Hungary
}

Contributions: (I) Conception and design: A Nemes; (II) Administrative support: N Ambrus; (III) Provision of study materials or patients: A Nemes, Á Kormányos, P Domsik, N Gyenes; (IV) Collection and assembly of data: Á Kormányos, K Vezendi, I Marton, Z Borbényi; (V) Data analysis and interpretation: Á Kormányos; (VI) Manuscript writing: All authors; (VII) Final approval of manuscript: All authors.

Correspondence to: Attila Nemes, MD, PhD, DSc, FESC. Department of Medicine, Faculty of Medicine, Albert Szent-Györgyi Clinical Center, University of Szeged, Semmelweis Street 8, P.O. Box 427, H-6725 Szeged, Hungary. Email: nemes.attila@med.u-szeged.hu.

Background: Hemophilia is an X-linked inherited disorder primarily affecting males, its major types are type A (deficiency in factor VIII) and B (deficiency in factor IX), and is considered to be the most common severe congenital coagulation factor deficiency. The present study was designed to test whether any differences in left ventricular (LV) rotational mechanics could be demonstrated between male patients with hemophilia and healthy controls using three-dimensional speckle-tracking echocardiography (3DSTE)derived virtual LV models.

Methods: The present study consisted of 17 patients with hemophilia, however, 3 patients were excluded due to insufficient image quality. In the remaining patient population, 12 patients had hemophilia A and 2 patients had hemophilia B (mean age: $42.2 \pm 18$.9 years, all males). The control group comprised 16 agematched healthy subjects ( $46.0 \pm 5.9$ years, all males).

Results: None of the routine two-dimensional echocardiographic data differ between patients with hemophilia and controls. None of the patients and controls showed $\geq$ grade 1 valvular regurgitations and had valvular stenoses. In one subject, the near absence of LV twist called as LV "rigid body rotation" could be detected, data of which were managed separately. While 3DSTE-derived apical LV rotation was 3.65 degrees, basal LV rotation proved to be 3.57 degrees leading to 0.08-degree LV apico-basal gradient suggesting counterclockwise LV "rigid body rotation". In the remaining patients, both LV apical rotation $(7.25 \pm 6.20$ vs. $10.39 \pm 4.16$ degrees, $\mathrm{P}<0.02)$ and $\mathrm{LV}$ twist $(10.24 \pm 5.60$ vs. $14.38 \pm 3.93$ degrees, $\mathrm{P}<0.003)$ showed significant impairment in patients with hemophilia.

Conclusions: LV rotational abnormalities are present in hemophilia with reduced LV apical rotation and twist.

Keywords: Three-dimensional (3D); speckle-tracking; echocardiography; hemophilia; left ventricular (LV); rotation; twist

Submitted Jan 08, 2021. Accepted for publication Jul 20, 2021.

doi: 10.21037/qims-21-30

View this article at: https://dx.doi.org/10.21037/qims-21-30 


\section{Introduction}

Hemophilia is an X-linked inherited disorder primarily affecting males, its major types are type A (deficiency in factor VIII) and B (deficiency in factor IX) and is considered to be the most common severe congenital coagulation factor deficiency (1). Its estimated prevalence is 17.1 cases $/ 100,000$ males for all severities of hemophilia A and 3.8 cases/100,000 males for all severities of hemophilia B (2). Together with atrial fibrillation, there is a possible higher risk for coronary artery disease (CAD) in hemophilia and its incidence is increasing due to the fact that life expectancy of hemophilia patients approximates that of the general population $(3,4)$. However, CAD mortality in hemophilia is lower compared to that of the general population possibly due to the protective effect on thrombus formation of the existing hypocoagulable state (4). Outcome of treatment for cardiovascular disease is similar to that in the general population in hemophilia (5). Moreover, no differences in cardiovascular comorbidities and their earlier onset could be demonstrated in hemophilia A compared to controls (6). Although lot of facts are known about cardiovascular diseases and the risk in hemophilia, no clinical data are available about hemophilia-related potential changes in myocardial mechanics.

Myocardial mechanics is highly dependent not only on cellular dysfunction, but also on left ventricular (LV) hypertrophy, fibrosis and wall stress (7). Hemostatic mechanisms are altered in hemophilia, which plays a major role in maintaining the structural and functional integrity of the vascular system, theoretically having effects on myocardial mechanics as well via changing wall stress (shear stress) (8). In all disorders in which any of these parameters are affected, theoretically myocardial mechanics could change. $\mathrm{LV}$ rotational mechanics is an important part of the $\mathrm{LV}$ pumping function showing early alterations in several disorders $(9,10)$. Three-dimensional (3D) speckle-tracking echocardiography (3DSTE) is a new clinical tool with the ability of non-invasive analysis of $\mathrm{LV}$ rotational mechanics using digitally acquired 3D "echocloud" to create a virtual cast of the LV (11). The present study was designed to test whether any differences in $\mathrm{LV}$ rotational mechanics could be demonstrated between male patients with hemophilia and healthy controls using 3DSTE-derived virtual LV model.

\section{Methods}

\section{Patient population}

The present study consisted of 17 patients with hemophilia, who were recruited on voluntary bases prospectively from the outpatient clinic of our tertiary Hematology Division, Department of Medicine, University of Szeged, Hungary. None of them had any known cardiovascular disorder. Due to insufficient image quality, 3 patients with hemophilia were excluded. In the remaining 14 patient population, 12 patients had hemophilia A and 2 patients had hemophilia B (mean age: $42.2 \pm 18.9$ years, all males). From cardiovascular risk factors, 3 patients had hypertension, 2 patients showed hyperlipidaemia and 1 subject had type 2 diabetes mellitus. One patient was obese, smoking was present in 2 cases. All above mentioned risk factors were managed with mono- or combined therapy. Although none of the subjects had any known cardiovascular disease, hepatitis $\mathrm{C}$ virus (HCV) positivity was present in 8 subjects and hemophilic arthropathy was diagnosed in 6 patients. Diagnosis was established in infant age in all cases. Symptoms were mild in 6 cases and severe in 8 subjects. Factor level was below $1 \%$ in 7 cases and $4 \%$ in 2 cases, $6 \%$ in 1 case, $9 \%$ in 2 cases, $10 \%$ in 1 case, $29 \%$ in 1 case, respectively. Therapy was based on demand in 7 patients and was prophylactic in 7 subjects. The mean dose of factors was between 1,000-6,000 U/week for each patient. The control group comprised 16 age-matched healthy subjects (46.0 \pm 5.9 years, all males). A subject was considered to be healthy, if he/she had no symptoms or cardiovascular risk factors, no history of chronic disease or medication use, had a negative physiological examination and routine electrocardiography (ECG) and echocardiography showing normal results.

Complete two-dimensional (2D) Doppler echocardiographic examination and 3DSTE were performed in all patients with hemophilia and controls by the same sonographer (ÁK). The presented work is a part of the Motion Analysis of the heart and Great vessels bY three-dimensionAl speckle-tRacking echocardiography in Pathological cases (MAGYAR-Path) Study, which aimed to assess diagnostic and prognostic value of 3DSTE-derived LV rotational parameters among others in different disorders including hemophilia ("magyar" means "Hungarian" in Hungarian language). The local institutional ethical committee approved the study, which complied with the ethical guidelines set in the 1975 Declaration of Helsinki (and updated versions) and all patients and controls gave informed consent.

\section{D Doppler echocardiography}

Routine echocardiography was performed using a Toshiba 


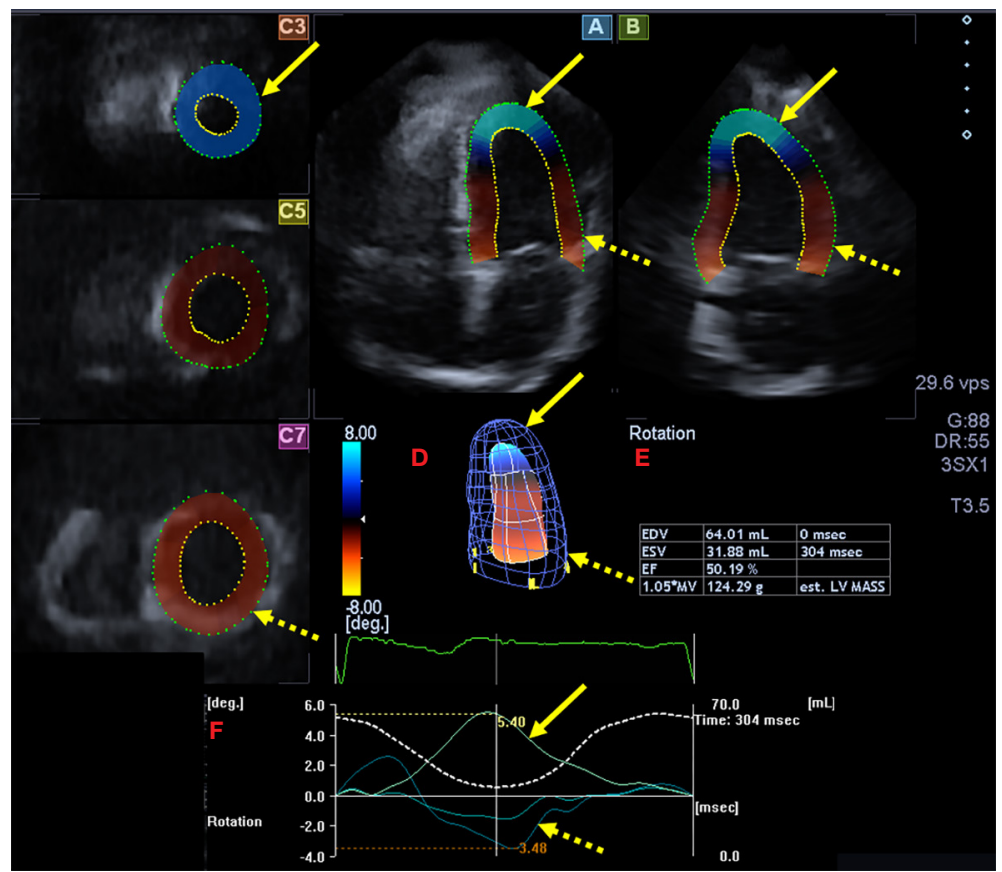

Figure 1 Analysis of a three-dimensional (3D) echocardiographic dataset: apical four-chamber view (A), apical two-chamber view (B) and apical (C3), mid-ventricular (C5), and basal LV (C7) short-axis views. A virtual 3D cast of the LV (red D), LV volumetric data respecting the cardiac cycle (red E), LV rotational curves (lines) and time-LV volume changes (dashed line) during the cardiac cycle (red F) are presented in a hemophilia patient with normal directions of LV rotational curves. Yellow arrow indicates maximum counterclockwise LV apical rotation, while dashed yellow arrow indicates maximum clockwise LV basal rotation. LV, left ventricle; EDV, end-diastolic volume; ESV, end-systolic volume; EF, ejection fraction.

Artida $^{\mathrm{TM}}$ echocardiography device (Toshiba Medical Systems, Tokyo, Japan). 2D grayscale harmonic images were acquired with a broadband $1-5 \mathrm{MHz}$ PST-30SBP phased-array transducer positioned in the left lateral position. Chamber quantification and LV ejection fraction (LVEF) measurements were performed in accordance with the guidelines. Relative wall thickness was measured as: interventricular septum (IVS) thickness + posterior wall (PW) thickness divided by LV diastolic diameter (12). Potential valvular heart diseases were evaluated with Doppler echocardiography.

\section{DSTE}

The same Toshiba Artida ${ }^{\mathrm{TM}}$ echocardiography device (Toshiba Medical Systems, Tokyo, Japan) equipped with a PST-25SX matrix-array transducer (Toshiba Medical Systems, Tokyo, Japan) with 3D capability was used for measurements (11). The apical window was used to acquire six wedge-shaped sub-volumes during a single breath-hold to create full volume 3D datasets. 3D Wall Motion Tracking software version 2.7 (Toshiba Medical Systems, Tokyo, Japan) was used for offline analysis of data. The software automatically selected several longand short-axis views at end-diastole from the 3D datasets acquired digitally. Regional LV rotations were defined as circumferential rotation around the long-axis of apical and basal segments of the LV during systole (in degrees). LV rotational mechanics were evaluated by the measurement of the following parameters (Figure 1) $(11,13)$ :

* LV basal (defined as the degree of clockwise rotation of LV basal myocardial segments) and apical (defined as the degree of counter-clockwise rotation of LV apical myocardial segments) rotation;

* LV twist (defined as the net difference between LV basal and apical rotations);

* Time to peak degree of LV twist from the start of the cardiac cycle;

* If apical and basal LV rotations were in the same 


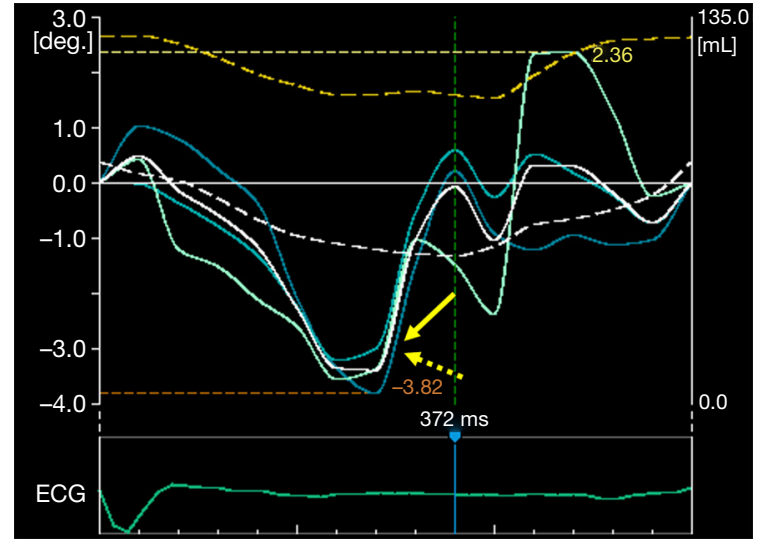

Figure 2 Demonstration of left ventricular (LV) rotational curves in a hemophilia patient with LV "rigid body rotation". All curves are in the same clockwise direction within almost the same amplitude. Yellow arrow indicates maximum (reversed) clockwise $\mathrm{LV}$ apical rotation, while dashed yellow arrow indicates maximum clockwise LV basal rotation.

clockwise or counterclockwise direction (which phenomenon is called as LV "rigid body rotation", LV-RBR), only LV apico-basal gradient could be calculated (maximum LV apical rotation minus maximum $\mathrm{LV}$ basal rotation) due to absence of $\mathrm{LV}$ twisting mechanics (Figure 2) $(13,14)$.

* Using the same 3D LV cast, LV longitudinal strain, the most frequently used LV strain parameter was also calculated.

\section{Statistical analysis}

Variables were presented as mean \pm standard deviation or frequencies and percentages (\%). Normality of distribution was assessed by Shapiro-Wilks test, while homogeneity of variances was tested by Levene's test. In case of normally distributed datasets, Student's $t$-test was performed, in case of not-normally distributed datasets, MannWhitney Wilcoxon test was used. GPower 3.1.9 Software (Heinrich-Heine Universität, Düsseldorf, Germany) was used to calculate power: in the presence of effect size: 0.8 , alpha: 0.04 , power: 0.8 , the minimum group size is $\mathrm{n}=13$. Intraobserver and interobserver variability were assessed by intraclass correlation coefficient (ICC) determination. Group comparisons were performed by Student's $t$-test and Fisher's exact test, when appropriate. Two-tailed $\mathrm{P}$ value $<0.05$ was used to establish statistical significance. MedCalc software was used in all statistical analyses (MedCalc, Inc., Mariakerke, Belgium).

\section{Results}

\section{Clinical and 2D Doppler echocardiographic data}

Mean systolic and diastolic blood pressure $(124.2 \pm 3.5$ vs . $123.5 \pm 2.9 \mathrm{mmHg}, \mathrm{P}=\mathrm{ns})$, heart rate $(72 \pm 7$ vs. $77 \pm 8 \mathrm{bpm}$, $\mathrm{P}=\mathrm{ns})$ and cardiac output $(5.9 \pm 0.6$ vs. $5.6 \pm 0.6 \mathrm{~L} / \mathrm{min}, \mathrm{P}=\mathrm{ns})$ did not show significant difference between patients with hemophilia and controls. Routine 2D echocardiographic data did not differ either (Table 1). None of the patients and controls showed $\geq$ grade 1 valvular regurgitations or had valvular stenoses.

\section{DSTE data}

In one subject, the near absence of LV twist called as LVRBR could be detected, data of this subject was managed separately. While apical LV rotation was 3.65 degrees, basal $\mathrm{LV}$ rotation proved to be 3.57 degrees leading to 0.08 -degree LV apico-basal gradient suggesting counterclockwise LVRBR (Figure 2). In the remaining 13 patients, both LV apical rotation $(7.25 \pm 6.20$ vs. $10.07 \pm 3.92$ degrees, $\mathrm{P}<0.02)$ and $\mathrm{LV}$ twist $(10.24 \pm 5.60$ vs. $14.41 \pm 4.26$ degrees, $\mathrm{P}<0.003)$ showed significant impairment in patients with hemophilia (Table 2).

\section{Reproducibility of 3DSTE-derived LV rotational parameters}

Intraobserver ICCs were $0.86,0.81$ and 0.82 for basal and apical $\mathrm{LV}$ rotations and $\mathrm{LV}$ twist, respectively. Interobserver ICCs proved to be $0.83,0.78$, and 0.80 for the same parameters, respectively.

\section{Discussion}

3DSTE is one of the most recent developments in cardiovascular imaging with capability of virtual 3D-modelbased volumetric and functional assessment of heart chambers and valvular annuli $(11,15,16)$. It provides a noninvasive, fast and easy-to-learn opportunity to perform 3D analysis of the atria and ventricles. While mathematical formulas are used for $\mathrm{LV}$ measurements during $\mathrm{M}$-mode and $2 \mathrm{D}$ echocardiography, an accurate $3 \mathrm{D}$ cast of the $\mathrm{LV}$ is created in the course of ECG-gated 3D endocardial tracking during $3 \mathrm{DSTE}$ leading to a true volumetric 
Table 1 Two-dimensional echocardiographic data of hemophilia patients and controls

\begin{tabular}{|c|c|c|}
\hline Parameters & Controls & Hemophilia patients \\
\hline LV end-diastolic diameter (mm) & $48.4 \pm 4.0$ & $50.5 \pm 3.2$ \\
\hline LV end-diastolic volume (mL) & $112.0 \pm 24.0$ & $123.0 \pm 18.6$ \\
\hline LV end-systolic diameter (mm) & $32.3 \pm 2.7$ & $31.9 \pm 3.0$ \\
\hline LV stroke volume (mL) & $73.2 \pm 7.9$ & $82.1 \pm 9.5$ \\
\hline Interventricular septum (mm) & $9.6 \pm 1.2$ & $9.9 \pm 1.0$ \\
\hline LV posterior wall (mm) & $9.5 \pm 1.1$ & $9.8 \pm 1.0$ \\
\hline LV length $(\mathrm{cm})$ & $9.4 \pm 1.6$ & $9.3 \pm 1.7$ \\
\hline$E(\mathrm{~cm} / \mathrm{s})$ & $70.4 \pm 19.6$ & $72.6 \pm 14.5$ \\
\hline$A(\mathrm{~cm} / \mathrm{s})$ & $61.2 \pm 15.7$ & $66.3 \pm 13.2$ \\
\hline$E / A$ & $1.20 \pm 0.36$ & $1.05 \pm 0.29$ \\
\hline
\end{tabular}

LA, left atrium; LV, left ventricular; E, early diastolic transmitral flow velocity; A, late diastolic transmitral flow velocity.

Table 2 Three-dimensional speckle-tracking echocardiography-derived left ventricular volumetric and rotational parameters in hemophilia patients and healthy controls

\begin{tabular}{lcc}
\hline Parameters & Controls & Hemophilia patients without LV-RBR \\
\hline Left ventricular volumetric parameters & & $81.6 \pm 23.9$ \\
LV-EDV $(\mathrm{mL})$ & $86.9 \pm 29.6$ & $39.1 \pm 4.1$ \\
LV-ESV $(\mathrm{mL})$ & $39.9 \pm 11.4$ & $51.9 \pm 4.1^{*}$ \\
LV-EF $(\%)$ & $56.5 \pm 5.6$ & $-2.99 \pm 2.16$ \\
Left ventricular rotational parameters & & $7.25 \pm 6.20^{*}$ \\
Basal LV rotation (degree) & $-3.99 \pm 2.20$ & $10.24 \pm 5.60^{*}$ \\
Apical LV rotation (degree) & $10.39 \pm 4.16$ & $412 \pm 181$ \\
LV twist (degree) & $14.38 \pm 3.93$ & $-15.3 \pm 3.8$ \\
Time of peak LV twist (ms) & $303 \pm 61$ & $-15.8 \pm 2.1$ \\
LV longitudinal strain $(\%)$ & & \\
\hline
\end{tabular}

${ }^{*}, \mathrm{P}<0.05$ vs. controls. EDV, end-diastolic volume; ESV, end-systolic volume; EF, ejection fraction; LV, left ventricular; RBR, rigid body rotation.

chamber quantification (17). It is known that 3DSTEderived LVEF is somewhat lower compared to M-mode or 2D echocardiography-derived values due to underestimated $\mathrm{LV}$ volumetric parameters, where EDV is more affected than ESV resulting in a lower 3DSTE-derived LVEF
$(18,19)$. Over volumetric measurements, quantitative features of contractility of heart chamber walls represented by $L V$ strains could also be measured in certain directions (radial, longitudinal and circumferential) in the $3 \mathrm{D}$ space using the same $L V$ cast $(11,15-17)$. 
Moreover, there is a complex movement of the $\mathrm{LV}$ during the cardiac cycle called LV rotational mechanics, which could be analysed in detail by the recently developed 3DSTE. In general, the base of the LV rotates in clockwise direction, while the LV apex rotates in counterclockwise direction in systole, which is followed by rapid untwisting in diastole in normal circumstances $(9,10)$. This sort of special and sensitive "towel-wringing"-like LV motion is called LV twist and it is responsible for remarkable part of the ejection. Physiologically, it is based on the helical arrangement of the myocardial fibers: while subendocardial myocardial fibers are right-handed, subepicardial ones are left-handed with dominant effects on $L V$ rotational mechanics due to their larger radius $(9,10)$. $L V$ rotational mechanics seem to be sensitive movement that are affected by aortic elasticity and stiffness, balance of contraction of subendocardium and subepicardium, orientation of myocardial fibers and degree of myocardial contraction and relaxation even in healthy subjects $(9,13)$. Calculation of 2D echocardiography-derived $\mathrm{LV}$ twist is not suggested by the most recent guidelines due to the fact, that $\mathrm{LV}$ twisting mechanism is a $3 \mathrm{D}$ motion of the $\mathrm{LV}$, therefore its $2 \mathrm{D}$ projected measurement would be far from the reality $(9,20)$. Therefore, 3DSTE, which is able to measure the exact degrees of $\mathrm{LV}$ rotation of each $\mathrm{LV}$ segments/regions and global LV twist from a single acquisition, seems to be the optimal solution.

The most important finding of the present study is that significant 3DSTE-derived LV rotational abnormalities could be demonstrated in patients with hemophilia. Although a small number of patients were examined, reduced apical LV rotation and twist were found in hemophilia. Moreover, one patient showed LV-RBR. The correct explanation is not obvious, but decreased $\mathrm{LV}$ twist and apical rotation should be considered as a fine compensational mechanism related to hemophilia-related hemostatic changes and related alterations in wall stress/ shear stress. It is strengthened by a recent study from the MAGYAR-Path Study, where only certain regional LV circumferential strains (CSs) proved to be reduced in hemophilia, global and mean segmental LV strains and other regional LV strains did not differ between patients with hemophilia and matched controls (21). Due to known relationship between LV-CS and LV twist, fine settings of $\mathrm{LV}$ mechanics via $\mathrm{LV}$ apical rotation could be theorized due these abnormalities in hemophilia. However, other factors like vascular functional alterations, the effects of concomitant cardiovascular risk factors, or other factors could also not be excluded $(4,8,22)$. According to recent findings, hypertension was found to be a frequent finding in hemophilia with some increase in septal thickness and changes in diastolic function (23). Forty-three percent of our patients had hypertension, which is known to be associated with increased LV twist, which further strengthen our theories (24). In a recent study, children with severe hemophilia A showed higher arterial stiffness, and myocardial performance index, whereas the ejection time was shorter than in the control group (22). Similar alterations in LV rotational mechanics could be detected in other hematological disorders as well including hypereosinophilic syndrome and amyloidosis with larger ratio of patients with LV-RBR $(25,26)$. However, further studies are warranted in a larger patient population to confirm our findings.

\section{Limitation section}

The following important limitations should be considered when interpreting the results. Hemophilia is a rare disease, therefore only limited number of patients could be collected and involved in the study from the tertiary center for patients with hematological disorders of our university responsible for the treatment of South-East Hungary. The present study aimed to analyse 3DSTE-derived LV rotational mechanics in hemophilia. Neither chambers other than the LV, nor LV strains featuring $L V$ contractility were aimed to be assessed by 3DSTE (11). Several technical limitations are known to affect 3DSTE including low temporal and spatial resolution $(11,15,16)$. Some adult hemophilia patients had risk factors and/or were treated with factor replacement therapy to prevent bleeding, which could theoretically have effects on findings.

\section{Conclusions}

$\mathrm{LV}$ rotational abnormalities are present in hemophilia with reduced $L V$ apical rotation and twist.

\section{Acknowledgments}

Funding: None.

\section{Footnote}

Conflicts of Interest: All authors have completed the ICMJE uniform disclosure form (available at https://dx.doi. org/10.21037/qims-21-30). AN serves as an unpaid editorial 
board member of Quantitative Imaging in Medicine and Surgery. The other authors have no conflicts of interest to declare.

Ethical Statement: The authors are accountable for all aspects of the work in ensuring that questions related to the accuracy or integrity of any part of the work are appropriately investigated and resolved. The study was conducted in accordance with the Declaration of Helsinki (as revised in 2013). The study was approved by institutional ethics committee of the University of Szeged (NO.: $71 / 2011$ ) and informed consent was taken from all the patients.

Open Access Statement: This is an Open Access article distributed in accordance with the Creative Commons Attribution-NonCommercial-NoDerivs 4.0 International License (CC BY-NC-ND 4.0), which permits the noncommercial replication and distribution of the article with the strict proviso that no changes or edits are made and the original work is properly cited (including links to both the formal publication through the relevant DOI and the license). See: https://creativecommons.org/licenses/by-nc-nd/4.0/.

\section{References}

1. Zimmerman B, Valentino LA. Hemophilia: in review. Pediatr Rev 2013;34:289-94; quiz 295.

2. Iorio A, Stonebraker JS, Chambost H, Makris M, Coffin D, Herr C, Germini F; Data and Demographics Committee of the World Federation of Hemophilia. Establishing the Prevalence and Prevalence at Birth of Hemophilia in Males: A Meta-analytic Approach Using National Registries. Ann Intern Med 2019;171:540-6.

3. Böhmert S, Schubert R, Fichtlscherer S, Alesci S, Miesbach W. Endothelial Function in Patients with Severe and Moderate Haemophilia A and B. Hamostaseologie 2019;39:195-202.

4. Tuinenburg A, Mauser-Bunschoten EP, Verhaar MC, Biesma DH, Schutgens RE. Cardiovascular disease in patients with hemophilia. J Thromb Haemost 2009; 7:247-54.

5. Ferraris VA, Boral LI, Cohen AJ, Smyth SS, White GC 2nd. Consensus review of the treatment of cardiovascular disease in people with hemophilia A and B. Cardiol Rev 2015;23:53-68.

6. Humphries TJ, Rule B, Ogbonnaya A, Eaddy M, Lunacsek O, Lamerato L, Pocoski J. Cardiovascular comorbidities in a United States patient population with hemophilia A: A comprehensive chart review. Adv Med Sci 2018;63:329-33.

7. Bianco CM, Farjo PD, Ghaffar YA, Sengupta PP. Myocardial Mechanics in Patients With Normal LVEF and Diastolic Dysfunction. JACC Cardiovasc Imaging 2020;13:258-71.

8. Rasche H. Haemostasis and thrombosis: an overview. Eur Heart J Suppl 2001;3:Q3-7.

9. Nakatani S. Left ventricular rotation and twist: why should we learn? J Cardiovasc Ultrasound 2011;19:1-6.

10. Nemes A, Kalapos A, Domsik P, Forster T. Left ventricular rotation and twist of the heart. Clarification of some concepts. Orv Hetil 2012;153:1547-51.

11. Nemes A, Kalapos A, Domsik P, Forster T. Threedimensional speckle-tracking echocardiography -- a further step in non-invasive three-dimensional cardiac imaging. Orv Hetil 2012;153:1570-7.

12. Lang RM, Badano LP, Mor-Avi V, Afilalo J, Armstrong A, Ernande L, Flachskampf FA, Foster E, Goldstein SA, Kuznetsova T, Lancellotti P, Muraru D, Picard MH, Rietzschel ER, Rudski L, Spencer KT, Tsang W, Voigt JU. Recommendations for cardiac chamber quantification by echocardiography in adults: an update from the American Society of Echocardiography and the European Association of Cardiovascular Imaging. Eur Heart J Cardiovasc Imaging 2015;16:233-70.

13. Nemes A, Kalapos A, Domsik P, Lengyel C, Orosz A, Forster T. Correlations between echocardiographic aortic elastic properties and left ventricular rotation and twist-insights from the three-dimensional speckle-tracking echocardiographic MAGYAR-Healthy Study. Clin Physiol Funct Imaging 2013;33:381-5.

14. van Dalen BM, Caliskan K, Soliman OI, Nemes A, Vletter WB, Ten Cate FJ, Geleijnse ML. Left ventricular solid body rotation in non-compaction cardiomyopathy: a potential new objective and quantitative functional diagnostic criterion? Eur J Heart Fail 2008;10:1088-93.

15. Urbano-Moral JA, Patel AR, Maron MS, Arias-Godinez JA, Pandian NG. Three-dimensional speckle-tracking echocardiography: methodological aspects and clinical potential. Echocardiography 2012;29:997-1010.

16. Ammar KA, Paterick TE, Khandheria BK, Jan MF, Kramer C, Umland MM, Tercius AJ, Baratta L, Tajik AJ. Myocardial mechanics: understanding and applying threedimensional speckle tracking echocardiography in clinical practice. Echocardiography 2012;29:861-72.

17. Nemes A, Forster T. Recent echocardiographic examination of the left ventricle - from M-mode to 3D 
speckle-tracking imaging. Orv Hetil 2015;156:1723-40.

18. Kormányos Á, Kalapos A, Domsik P, Gyenes N, Lengyel C, Nemes A. Normal reference values of left ventricular volumetric parameters in healthy adults-real-life singlecenter experience from the three-dimensional speckletracking echocardiographic MAGYAR-Healthy Study. Quant Imaging Med Surg 2021;11:1496-503.

19. Driessen MMP, Kort E, Cramer MJM, Doevendans PA, Angevaare MJ, Leiner T, Meijboom FJ, Chamuleau SAJ, Sieswerda GTj. Assessment of LV ejection fraction using real-time $3 \mathrm{D}$ echocardiography in daily practice: direct comparison of the volumetric and speckle tracking methodologies to CMR. Neth Heart J 2014;22:383-90.

20. Voigt JU, Pedrizzetti G, Lysyansky P, Marwick TH, Houle H, Baumann R, Pedri S, Ito Y, Abe Y, Metz S, Song JH, Hamilton J, Sengupta PP, Kolias TJ, d'Hooge J, Aurigemma GP, Thomas JD, Badano LP. Definitions for a common standard for 2D speckle tracking echocardiography: consensus document of the EACVI/ ASE/Industry Task Force to standardize deformation imaging. J Am Soc Echocardiogr 2015;28:183-93.

21. Nemes A, Kormányos Á, Vezendi K, Marton I, Borbényi Z. Left ventricular deformation in haemophilia (from the
MAGYAR-Path Study). Rev Port Cardiol 2021. [In press].

22. Özdemir ZC, Köşger P, Uçar B, Bör Ö. Myocardial functions, blood pressure changes, and arterial stiffness in children with severe hemophilia A. Thromb Res 2020;189:102-7.

23. Amoozgar H, Fath M, Jooya P, Karimi M. Evaluation of Heart Function in Patients With Hemophilia. Clin Appl Thromb Hemost 2017;23:374-8.

24. Gnakamene JB, Safar ME, Levy BI, Escoubet B. Left Ventricular Torsion Associated With Aortic Stiffness in Hypertension. J Am Heart Assoc 2018;7:007427.

25. Nemes A, Kormányos Á, Domsik P, Kalapos A, Ambrus N, Modok S, Borbényi Z, Marton I. Left ventricular rotational mechanics in hypereosinophilic syndrome-Analysis from the three-dimensional speckle-tracking echocardiographic MAGYAR-Path Study. Echocardiography 2019;36:2064-9.

26. Nemes A, Földeák D, Domsik P, Kalapos A, Sepp R, Borbényi Z, Forster T. Different patterns of left ventricular rotational mechanics in cardiac amyloidosisresults from the three-dimensional speckle-tracking echocardiographic MAGYAR-Path Study. Quant Imaging Med Surg 2015;5:853-7.
Cite this article as: Nemes A, Kormányos Á, Domsik P, Ambrus N, Gyenes N, Vezendi K, Marton I, Borbényi Z. Left ventricular rotational abnormalities in hemophiliainsights from the three-dimensional speckle-tracking echocardiographic MAGYAR-Path Study. Quant Imaging Med Surg 2022;12(2):886-893. doi: 10.21037/qims-21-30 